\title{
Next Generation Service Delivery Platforms
}

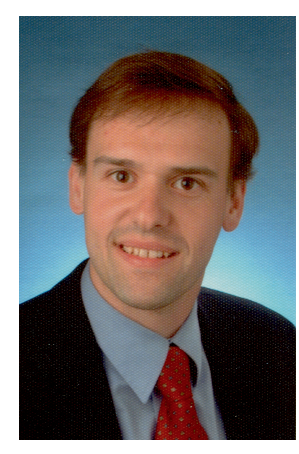

Wolfgang Kellerer

Lehrstuhl für Kommunikationsnetze Technische Universität München Arcisstr. 21

80333 München

Germany

wolfgang.kellerer@ tum.de

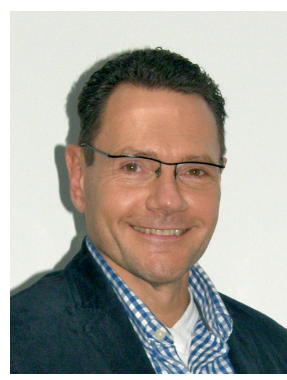

\section{Thomas Magedanz}

Technische Universität Berlin

FR 5-14

Franklinstr. 28/29

10587 Berlin

Germany

Thomas.magedanz@tu-berlin.de

Telecommunication has emerged as a cross-discipline technology penetrating almost all aspects of our everyday private and business life. This includes providing traditional telco-oriented services such as voice, video and data communication, the vastly increasing variety of web-based applications for smartphones and tablets as well as the support of emerging applications in the areas of automobile traffic, logistics, machine-to-machine communication, energy management, and smart cities.

Service Delivery Platforms (SDP) play a key role in the creation and deployment of innovative services in all disciplines. We refer to Service Delivery Platform for a network infrastructure that allows providing value-added services seamlessly on top of existing and emerging fixed and mobile communication networks. With the emergence of new network technologies and, in particular, with the adoption of Internet (namely IP based) technologies for telecommunication and media networks a first step has been achieved towards a unified service infrastructure providing a common transport protocol. However, more complex services as we see emerging today raise the need for more sophisticated platforms offering building blocks to serve the whole value chain of service provisioning. Still today, the area of SDP concepts and systems is highly fragmented and there exists no common approach for converged services or a single standard [1].

In view of the above and in order to foster the discussion on future Service Delivery Platforms, a new discussion forum has been founded in KuVS with the name "Next Generation Service Delivery Platforms (NGSDP)" in 2009 [2,3]. The objective of this forum is to foster the SDP community and prepare the ground for a consolidated view on the SDP related scientific research and industrial development.

Up to now, six expert meetings (Fachgespräche) have been held; three in Berlin (November 2009, organized by Fraunhofer FOKUS, October 2010, organized by Deutsche Telekom, and April 2012, organized by Fraunhofer FOKUS), two in Munich (May 2010, organized by Telefonica O2 and October 2011, organized by DOCOMO Communication Laboratories Europe), and one in Vienna (April 2011, organized by University of Vienna and Telecom Austria). The upcoming seventh meeting will be held in Zurich in October 2012. Each meeting has been attended by $30-50$ participants well balanced between industry and academia. Meeting themes have included service value chains, SDPs for future Internet, for mobile networks, for cloud computing and recently for machine-to-machine communication. For more information and for all slides presented see [3].

This special issue features six selected papers presented at the fourth and fifth Fachgespräch that took place in $\mathrm{Mu}-$ nich on "Advanced Service Delivery Platforms for Mobile Networks" and in Vienna on "Bringing SDPs to the Future Internet and Future Generation Networks", respectively. It follows a first special issue published in 2010 covering selected papers of earlier meetings [1].

The first two articles are addressing the provisioning and optimization of mobile video streaming applications. The third and the forth article target service delivery in cloud computing environments. The fifth article addresses resilient service provisioning for over the top service platforms. The last article proposes an API for the convergence of web and telco services. This selection of articles represents the diversity of the research field of Service Delivery Platforms ranging from resource and traffic management for optimal service delivery to APIs for application deployment targeting the "upper layers" of communication and information systems in terms of protocols, algorithms and APIs.

In the first article "Quality of Experience Management for YouTube: Clouds, Rain, and FoG” Tobias Hoßfeld et al. investigate the potential as well as the implementation challenges of QoE management in the Internet. The authors elaborate QoE management requirements for two complementary network scenarios (wireless mesh networks and global Internet delivery) and provide a QoE model for YouTube taking into account impairments like stalling and initial delay. Furthermore, the authors present two YouTube QoE monitoring approaches operating on the network and the end user level. Finally, they demonstrate how QoE can be dynamically optimized in both network scenarios with two exemplary concepts Aquarema and Forwarding on Gates (FoG), respectively.

The second article "Analysis of Managed and Over-theTop Streaming Services in Mobile Networks" by J. Eisl et al. aims for optimizing multimedia streaming in 3GPP mobile broadband networks (e.g. HSPA, LTE) in terms of user expe- 
rience and network resource utilization. The authors have carried out several field experiments to obtain knowledge about typical bandwidth consumption for the most popular overthe-top and managed video services. They detected that observed data rates apart from the video encoding and the specific content vary a lot between different client and server implementations.

The third article "Optimization of Elastic Cloud Brokerage Mechanisms for Future Telecommunication Service Environments" by K. Campowsky et al. addresses mechanism for dynamic allocation of compute and storage resources ondemand, commonly termed as "elastic cloud computing". The presented work focuses on enhanced cloud brokering mechanisms for telecommunication service platforms, enabling telecommunication service quality assurance, still optimizing cloud resources consumption, i.e. saving costs and energy. Furthermore this work shows that by combining cloud brokering mechanisms with standardized telecommunication service brokering mechanisms an even greater benefit for telecommunication service providers can be achieved.

M. Stecca et al. address fault tolerance in cloud computing in the fourth article "Experiments and analysis on Hypervisor-based Fault Tolerance in Virtualized Cloud Environments". After comparing different fault tolerance directions Infrastructure as a Service providers may follow, the paper focuses on Hypervisor-based Fault Tolerance. The analysis is based on a set of experiments on the VMware vSphere system. The authors include a description of how the VMware Fault Tolerance system works and a description of how the performance of different types of applications (in particular I/O-intensive and CPU-intensive) are affected by the inclusion of the Fault Tolerance mechanism.

The fifth article "Smooth Resilient Service Provision in Large Heterogeneous Networks" written by K. Panitzek et al. presents a first solution to the challenge of providing fault tolerant services, such as multiplayer online games, above fixed and mobile networks which are exposing Byzantine errors and malicious attacks. The authors combine support for Byzantine error correction, mobile code, smooth handover, and availability and performance improvements through replication and intelligent service placement.

In the sixth article, Joachim Zeiß et al. present a concept and implementation for an API called APSINT residing on a mobile phone to allow web applications to run telecommunication operator services without setting up a separate business-to-business relationship. In addition to a concept description and discussion, the authors describe their implementation and its evaluation.

We would like to thank all members of the Fachgespräch Next Generation Service Delivery Platforms and, in particular, the authors and reviewers of the special issue papers. We hope you enjoy reading the papers and hope to see you at some of the future workshops. New members are very welcome. For more information on the Fachgespräch, please contact the editors, visit the KuVS [2] or the Fachgespräch [3] web page or subscribe to the mailing list [4].

\section{References}

[1] W. Kellerer, T. Magedanz: Next Generation Service Delivery Platforms and Service Overlay Networks (Special Issue). PIK. Vol. 33, No. 1, 2010.

[2] http://www.kuvs.de/fachgespr/servicedelivery-platforms-ngsdp/.

[3] http://www.kuvs-ngsdp.org/index.html.

[4] Mailing List: sdp_group@kuvs-ngsdp.org. 